\title{
Design of planar millimeter-wave metallic structures for Wakefield acceleration
}

\author{
Liang Zhang • Wenlong He • Steven \\ Jamison · Colin G. Whyte - Kevin \\ Ronald • Alan D.R. Phelps • Adrian W. \\ Cross
}

Received: September 2018

\begin{abstract}
Linear accelerators operating at millimeter or sub-terahertz frequencies and short pulse duration have the advantages of lower power consumption and high repetition rate. In this paper planar metallic accelerating structures with different modes operating at $210 \mathrm{GHz}$ were designed. A tolerance study was also carried out to determine the sensitivities of the geometric parameters to the wakefield acceleration performance. The generated Wakefield was simulated using the beam parameter of the Compact Linear Advanced Research Accelerator (CLARA) test facility at Daresbury Laboratory. For a $55 \mathrm{MeV}$ single electron bunch with charge of $250 \mathrm{pC}$ and a bunch length of $0.27 \mathrm{~mm}(0.9 \mathrm{ps})$, an equivalent acceleration gradient of $20 \mathrm{MV} / \mathrm{m}$ was achieved in the simulation. The relatively modest acceleration gradient was limited by the charge in a single bunch. The acceleration gradient could be further improved by using a bunch train which has larger total bunch charge. From the simulation, the acceleration gradient of $100 \mathrm{MV} / \mathrm{m}$ can be generated when it is driven by a 10-bunch beam train.
\end{abstract}

Keywords planar metallic waveguide $\cdot$ Wakefield generation $\cdot$ THz acceleration acceleration structure

\section{Introduction}

The accelerators nowadays mainly operate at S-, C-, and X-bands. The acceleration gradient is one of the most important parameters for a linear accelerator, and it has always increased as development techniques have improved. For example, the SLAC (Stanford Linear Accelerator Center) S-band linac achieved $17 \mathrm{MV} / \mathrm{m}$ in the late 1960s [1]. The NLC (Next Linear Collider) operating in X-band achieved

This work was supported by STFC Cockcroft Institute Core Grant ST/P002056/1.

L. Zhang, W. He, C.G. Whyte, K. Ronald, A.D.R. Phelps, and A. W. Cross are with Department of Physics, SUPA, University of Strathclyde, Glasgow, G4 0NG, UK. S. Jamison is with Cockcroft Institute and the Department of Physics, University of Lancaster, a member of ASTeC. L. Zhang, C.G. Whyte, K. Ronald and A.W. Cross are with Cockcroft Institute, Sci-Tech Daresbury, Warrington WA4 4AD, U.K.

E-mail: liang.zhang@strath.ac.uk 
an acceleration gradient of $65 \mathrm{MV} / \mathrm{m}$ in the 1990s [2]. Currently, the CLIC (Compact Linear Collider) under development at CERN has achieved an acceleration gradient of $100 \mathrm{MV} / \mathrm{m}$ at X-band [3]. The increasing demand for higher acceleration gradient is driven by the requirements for the next generation synchrotron light sources [4].

Operating at millimeter or sub-terahertz frequencies and short pulse duration has a few advantages. One is that the acceleration gradient increases with the operating frequency at a fixed input power. For example, the acceleration gradient at $\mathrm{C}$ band $(6 \mathrm{GHz})$ can be $50 \mathrm{MV} / \mathrm{m}$ at a power of $1 \mathrm{GW}$ [5], $100 \mathrm{MV} / \mathrm{m}$ at X-band $(12 \mathrm{GHz})$ for a power of $50 \mathrm{MW}[6]$. while at W-band $(94 \mathrm{GHz})$, the acceleration gradient can be $100 \mathrm{MV} / \mathrm{m}$ with a power of $10 \mathrm{MW}$ using cavities of a similar Q. The power requirement is much smaller when operating at higher frequencies. It can be a significant benefit for high energy linear accelerators of the multi-km scale to reduce the overall power consumption. Also the microwave pulse length required in a higher frequency acceleration structure is shorter, which is helpful for the applications that require a higher repetition rate.

However it is still challenging to generate intense millimeter or terahertz radiation. Different techniques are under development, including the laser-driven or beam-driven methods $[7,8]$. And acceleration media like dielectric materials and plasma are used to improve the breakdown threshold to $1 \mathrm{GV} / \mathrm{m}$ to $10 \mathrm{GV} / \mathrm{m}$ range. For acceleration in dielectric materials, intense short pulse radiation in the terahertz frequency range can be used to accelerate short electron bunches. In the laser-driven dielectric method, the intense terahertz radiation is produced by the interaction of an ultra-short laser pulse with the eigenmode of a waveguide structure. While in the beam-driven method, the radiation is generated from the interaction between the high-relativistic short electron bunch and the waveguide structure.

The Dielectric and THz Acceleration (DATA) project at the Cockcroft Institute aims to realize the electron bunch acceleration driven using high power terahertz waves [9]. The research in this paper is one of the schemes, to investigate metallic structures driven by short electron bunches for Wakefield acceleration $[10,11]$.

In this paper, the design of a planar metallic acceleration structure operating at $210 \mathrm{GHz}$ is presented. The performance of both $2 \pi / 3$ and $4 \pi / 5$ modes are compared, and the Wakefield strength driven by the electron bunches of CLARA was studied by numerical simulation.

\section{Metallic Wakefield Structure}

The operating parameters of the metallic structure were chosen based on the CLARA facility, whose beam parameters are listed in Table 1. The CLARA beam of energy $55 \mathrm{MeV}$ that has the higher bunch charge of $250 \mathrm{pC}$ with a bunch length of about $0.27 \mathrm{~mm}(0.9 \mathrm{ps})$ will be used. The operating frequency of the $\mathrm{RF}$ photo-injector gun is $3 \mathrm{GHz}$, and the metallic structure was chosen to operate at its harmonic frequency. A high operating frequency, such as $420 \mathrm{GHz}$, would achieve high acceleration field strength. However, the smallest dimension of the structure was estimated to be about $0.1 \mathrm{~mm}$, which is challenging to achieve using the conventional machining techniques. To allow larger dimensions, a lower oper- 

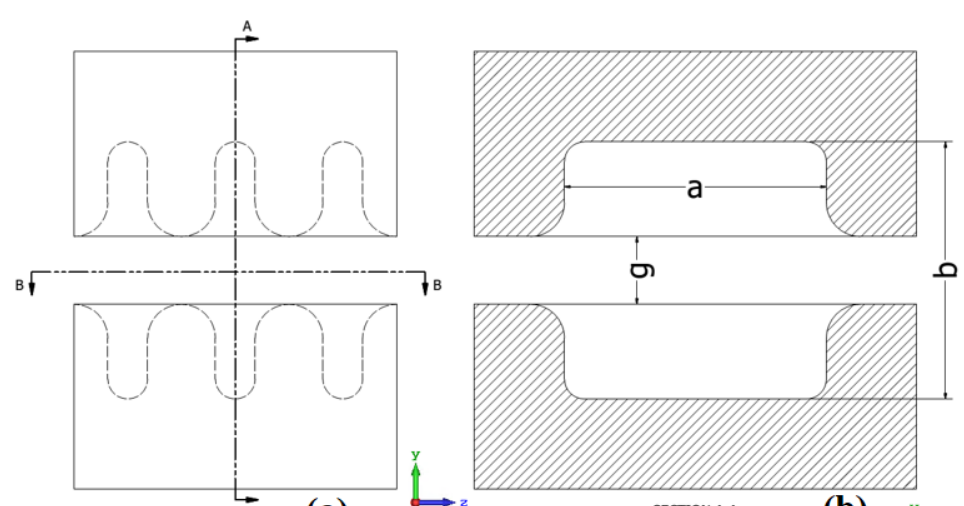

7 в

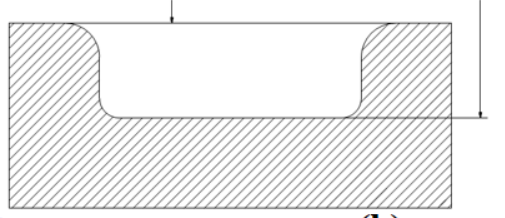

(a)

(b)
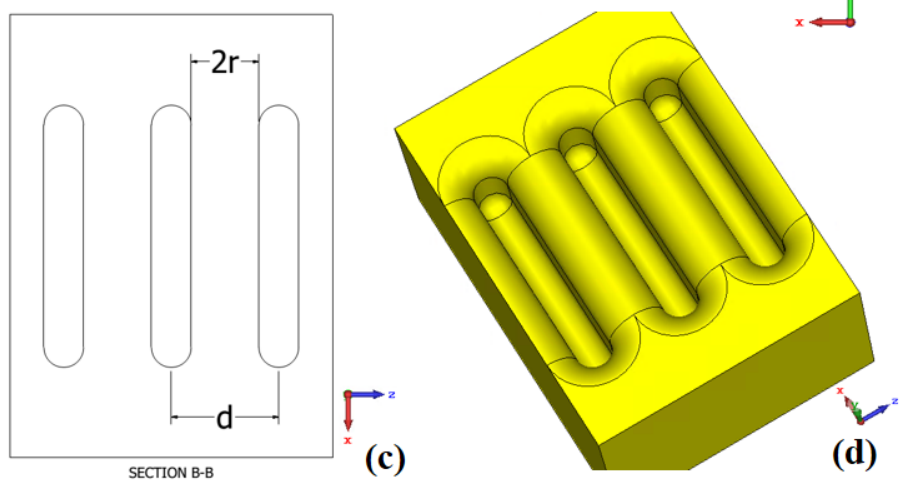

Fig. 1 Drawings of the planar metallic structure. (a) cross-section view of $Y Z$ plane, (b) cross-section view of $X Y$ plane, (c) cross-section view of $X Z$ plane, (d) 3D view of the half of cavity structure.

ating frequency of $210 \mathrm{GHz}$ was chosen, which corresponds to the 70th harmonic frequency of the photo-injector gun.

\begin{tabular}{lll}
\hline Parameters & Values & Values \\
\hline Bunch charge $Q$ & $100 \mathrm{pC}$ & $250 \mathrm{pC}$ \\
\hline Beam energy & $55 \mathrm{MeV}$ & $55 \mathrm{MeV}$ \\
\hline RMS beam size & $<0.1 \mathrm{~mm}$ & $0.1 \mathrm{~mm}$ \\
\hline Norm. emittance $(\mathrm{RMS})$ & $5 \mu \mathrm{m}$ & $10 \mu \mathrm{m}$ \\
\hline RMS bunch length $\sigma_{z}$ & $0.2 \mathrm{ps}$ & $0.9 \mathrm{ps}$ \\
\hline RMS Energy spread & $1.4 \%$ & $1.0 \%$ \\
\hline Frequency of photo-injector gun & $3 \mathrm{GHz}$ & $3 \mathrm{GHz}$ \\
\hline
\end{tabular}

Table 1 Beam parameters of CLARA. (RMS = Root-Mean-Square)

Among the various acceleration structures, the planar structure was chosen because it is easier to machine as compared with the ridged metallic structures. A planar structure was chosen, similar to the ones used in references [12-14], as shown in Fig. 1. The whole structure is composed of two halves and each half contains a half cavity. To avoid the electric field enhancement at the cavity edges, 
all the edges are chamfered. The biggest advantage of the structure is that the half cavity structure can be machined individually by Computer Numerical Controlled (CNC) micro-milling then assembled together. Therefore, it is able to significantly reduce the machining complexity and cost. As the structure is composed of two plates, it also has the advantage of being able to adjust the operating frequency by changing the gap distance $((g$ in Fig. 1$))$ between the two plates. CLARA has an RMS beam size of $0.1 \mathrm{~mm}$, therefore a gap distance of $0.3 \mathrm{~mm}$ was used to allow the electron bunch to pass through freely.

The acceleration structure was designed to operate in a traveling wave mode. To interact with the highly relativistic electron bunch, its dispersion curve needs to intersect with the beamline and the intersection point should be at the desired operating frequency of $210 \mathrm{GHz}$. Therefore the relationship between the phase advance $(\Theta)$ of each structure period $(d)$ and the operating frequency $(f)$ is

$$
2 \pi f=k_{z} v_{z} \approx \Theta c / d
$$

where $c$ is the speed of light. Normally the phase advance of each period can be chosen as $2 \pi / 3$. It gives a period of $0.48 \mathrm{~mm}$ at $210 \mathrm{GHz}$. From Fig. 1, it can be seen that each period contains both the cavity and the gap between the adjacent cavities. The wall thickness is twice the chamfer radius ( $r$ in Fig. 1$)$. The wall can not be too thin as it has to be mechanically strong so as not be deformed during the machining. Therefore the wall thickness of $0.3 \mathrm{~mm}$ was chosen. As a result, the cavity size becomes very small. To ease the fabrication requirements, it is possible to operate in a mode with a slightly larger phase shift, for example, $4 \pi / 5$, to give an extra $20 \%$ of the period length, which becomes $0.57 \mathrm{~mm}$.

The cavity size can be estimated from a simplified geometry. The gap distance between the two waveguide plates is small to cutoff the generated wakefield radiation. By ignoring the effect of the round corners at the cavity edges, the cavity shape can be approximated as a cubic shape. Its resonance frequency can be written as

$$
f=\frac{c}{2} \sqrt{(m / a)^{2}+(n / b)^{2}+(1 / \lambda)^{2}}
$$

where $m, n$ and $l$ are integers defining the mode, $a$ and $b$ represent the cavity size in the $X$ and $Y$ direction, as labeled in Fig. 1. $\lambda$ is the wavelength of the propagation wave, which can be calculated from the phase advance and the structure period. The TM modes are suitable for beam acceleration as it has a large $E_{z}$ component. $m=n=1$ is chosen as the $\mathrm{TM}_{11}$ mode has small $E_{x}$ and $E_{y}$ components at the center. Since the dimensions of the acceleration structure is small, it is reasonable to choose $a \approx b$. Therefore $a \approx 1.17 \mathrm{~mm}, \lambda \approx 1.43 \mathrm{~mm}$ can be obtained for both cases of $\Theta=2 \pi / 3$ and $\Theta=4 \pi / 5$.

After choosing the initial dimensions, CST microwave studio [15] was used to calculate the eigenfrequencies more accurately. The simulation model contained 3 periods for the $2 \pi / 3$ mode and 5 periods for $4 \pi / 5$ mode. A periodic boundary condition was used with a phase shift of $2 \pi$ and $4 \pi$, respectively, for the two modes. To achieve an eigen frequency that is exactly the same with the operating frequency, the dimensions of $a$ and $b$ were adjusted slightly. The final dimensions of the acceleration structure are listed in Table 2. The electric field patterns of both the eigenmodes $(2 \pi / 3$ and $4 \pi / 5)$ at YZ plane $(X=0)$ are shown in Fig. 2. 
Table 2 Dimensions of acceleration structure.

\begin{tabular}{|c|c|c|c|c|}
\hline \multirow{2}{*}{ Parameters } & \multicolumn{2}{|c|}{$2 \pi / 3$ mode } & \multicolumn{2}{|c|}{$4 \pi / 5$ mode } \\
\hline & Initial & Adjusted & Initial & Adjusted \\
\hline$a(\mathrm{~mm})$ & 1.17 & 1.16 & 1.17 & 1.16 \\
\hline$b(\mathrm{~mm})$ & 1.17 & 1.14 & 1.17 & 1.10 \\
\hline Period $d(\mathrm{~mm})$ & 0.48 & 0.48 & 0.57 & 0.57 \\
\hline Chamfer radius $r(\mathrm{~mm})$ & 0.15 & 0.15 & 0.15 & 0.15 \\
\hline Gap distance $p(\mathrm{~mm})$ & 0.3 & 0.3 & 0.3 & 0.3 \\
\hline
\end{tabular}

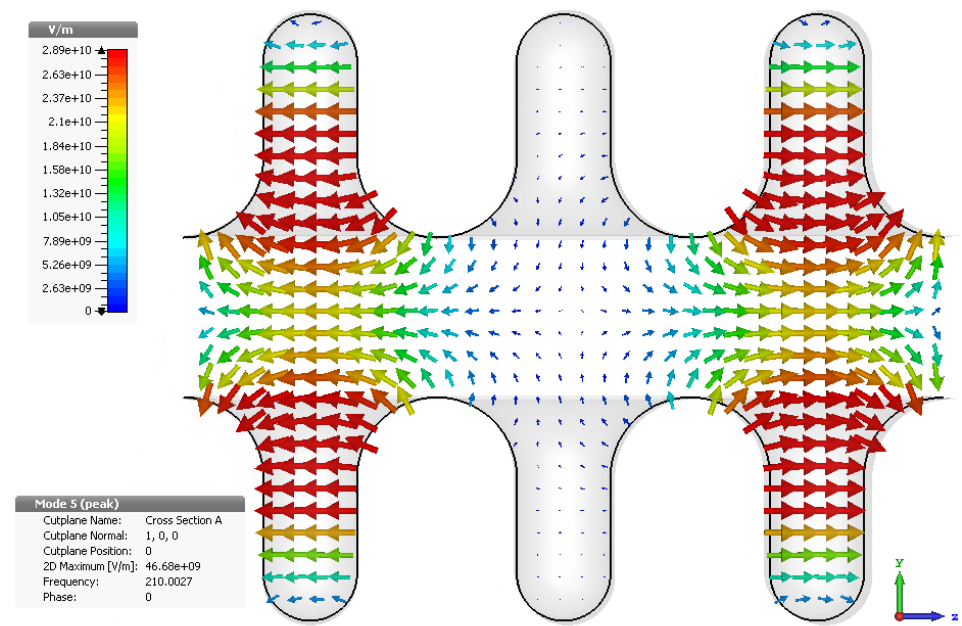

(a)

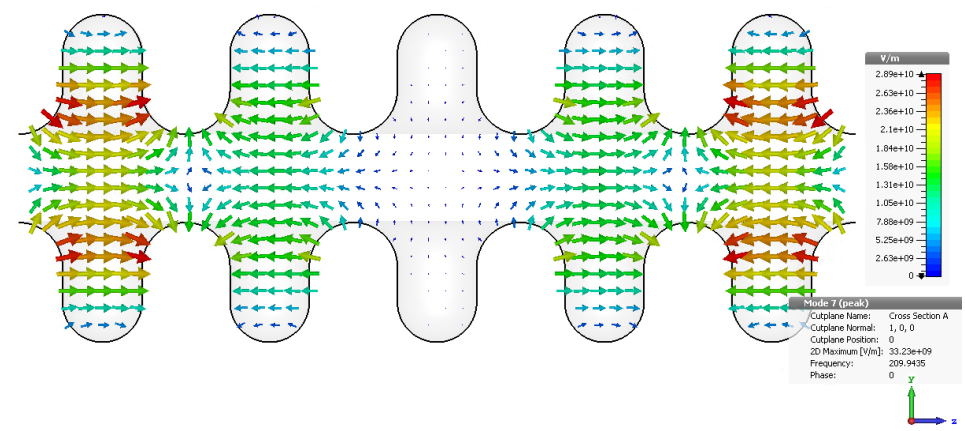

(b)

Fig. 2 Electric field patterns of the $2 \pi / 3$ (a) and $4 \pi / 5$ (b) modes.

The breakdown limit of the acceleration structure is determined by the field strength at the wall, which is shown in Fig. 3 for both the $2 \pi / 3$ and $4 \pi / 5$ modes. For the $2 \pi / 3$ mode, the peak electric field at the wall is $100 \mathrm{MV} / \mathrm{m}$ and the acceleration field strength on the cavity center is $49 \mathrm{MV} / \mathrm{m}$, when it is driven by an input power of $1 \mathrm{MW}$. For the $4 \pi / 5$ mode, the field strengths are slightly 


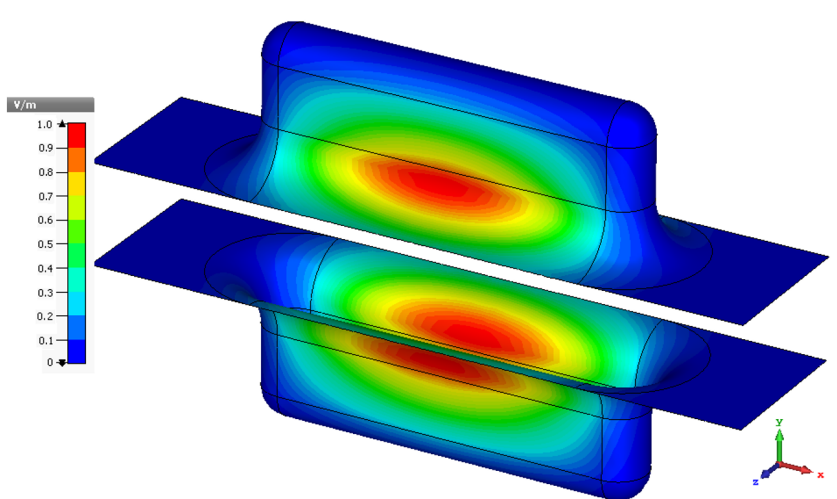

(a)

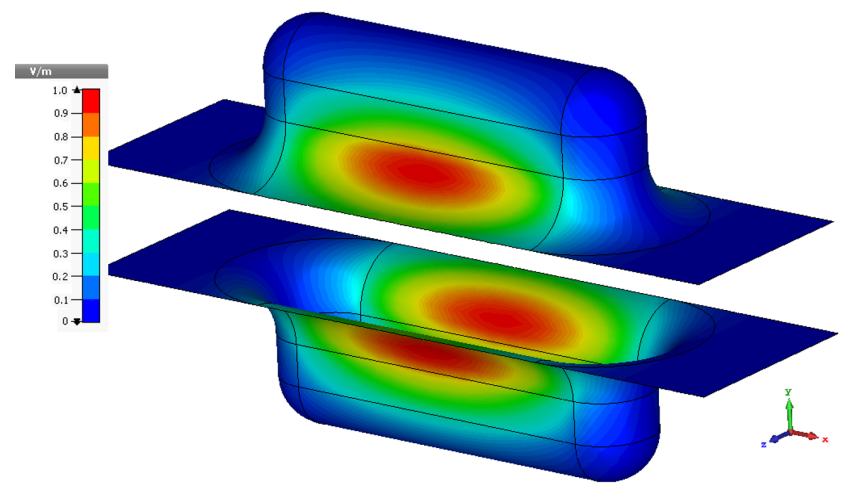

(b)

Fig. 3 Electric field patterns on the cavity walls of the $2 \pi / 3$ (a) and $4 \pi / 5$ (b) modes.

smaller, the peak field strength of the wall is $96 \mathrm{MV} / \mathrm{m}$ and the acceleration field strength is $44 \mathrm{MV} / \mathrm{m}$ at the center.

\section{TOLERANCE STUDY OF THE METALLIC WAKEFIELD STRUCTURE}

Parameters sweeps were used to study the effect of the key geometry parameters listed in Table 2 to determine the requirements of the machining tolerance, as well as the alignment requirement of the two half cavities. The results of the tolerance dependence of two of the most important parameters, eigenfrequency and shunt impedance are shown in Fig. 4 . The shunt impedance $R_{s}$ is defined by $U^{2} / 2 P_{d} . P_{d}$ and $U$ are the dissipated power and the stored energy in the simulated structure. They can be obtained from the results of the eigenmode solver. The higher the shunt impedance, the higher the acceleration gradient of the Wakefield structure can achieve.

Increasing the cavity dimensions, such as the $a, b$, period and gap distance would increase the cavity size, and reduce the eigenfrequency of the operating mode 


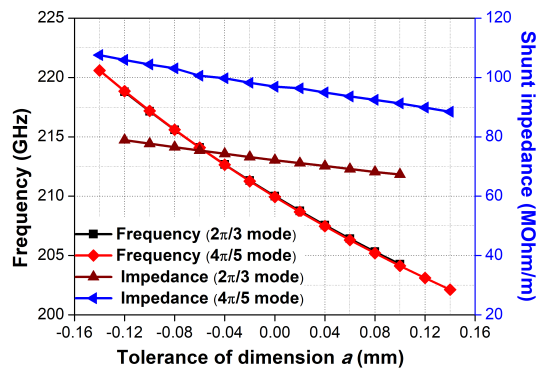

(a)

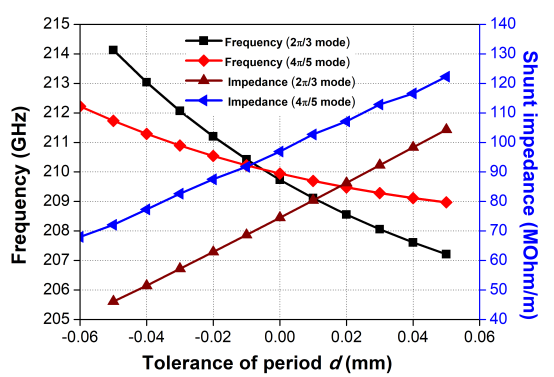

(c)

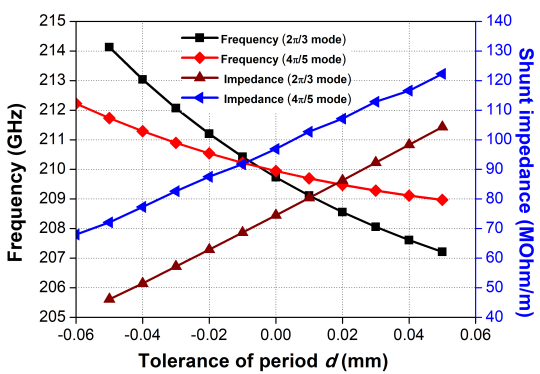

(e)

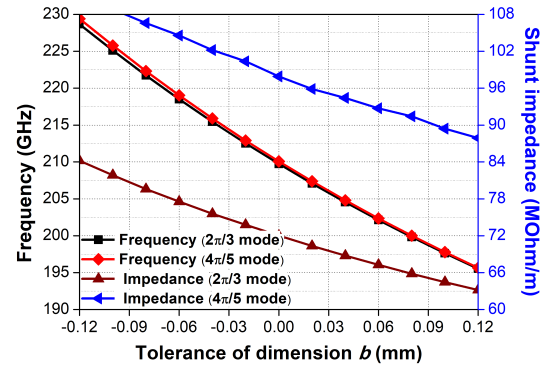

(b)

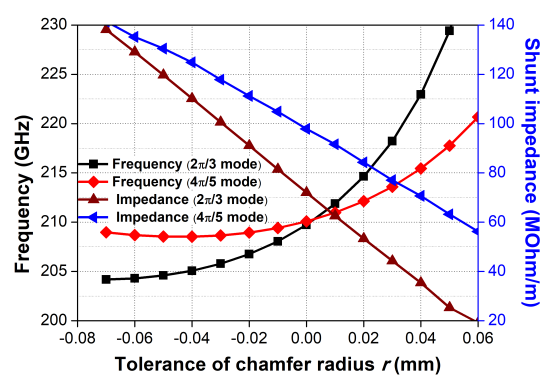

(d)

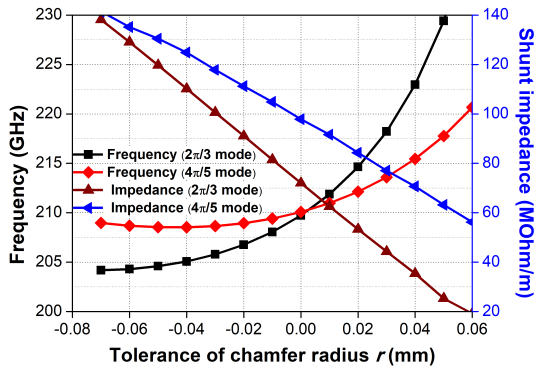

(f)

Fig. 4 Parameter scans results of the eigenmode solver, (a) the tolerance of dimension $a$, (b) the tolerance of dimension $b,(\mathrm{c})$ the tolerance of period $d,(\mathrm{~d})$ tolerance of the chamfer radius $r,(e)$ offset of the two plates and (f) the gap distance $g$.

accordingly. The sensitivity of dimension a was about $1.34 \mathrm{GHz}$ per $20 \mu \mathrm{m}$ for both the $2 \pi / 3$ and $4 \pi / 5$ modes. The eigenfrequencies were more sensitive to dimension $b$ and the variation was about $2.80 \mathrm{GHz}$ per $20 \mu \mathrm{m}$ in tolerance. In general, operating in the $2 \pi / 3$ mode was more sensitive to the dimension tolerance than the $4 \pi / 5$ mode, because it had smaller dimensions. The sensitivity of the eigenfrequencies for the $4 \pi / 5$ mode was about $0.6 \mathrm{GHz}$ at a $20 \mu \mathrm{m}$ tolerance on the period value. Increasing the period value helped to improve the shunt impedance, which was on an opposite trend as compared with dimensions $a$ and $b$.

It was found that when it was with minus tolerance, the variation of the chamfer radius $r$ did not change the eigenfrequency much, however, it had a stronger impact on the shunt impedance. A $20 \mu \mathrm{m}$ increment on the chamfer radius resulted in a 


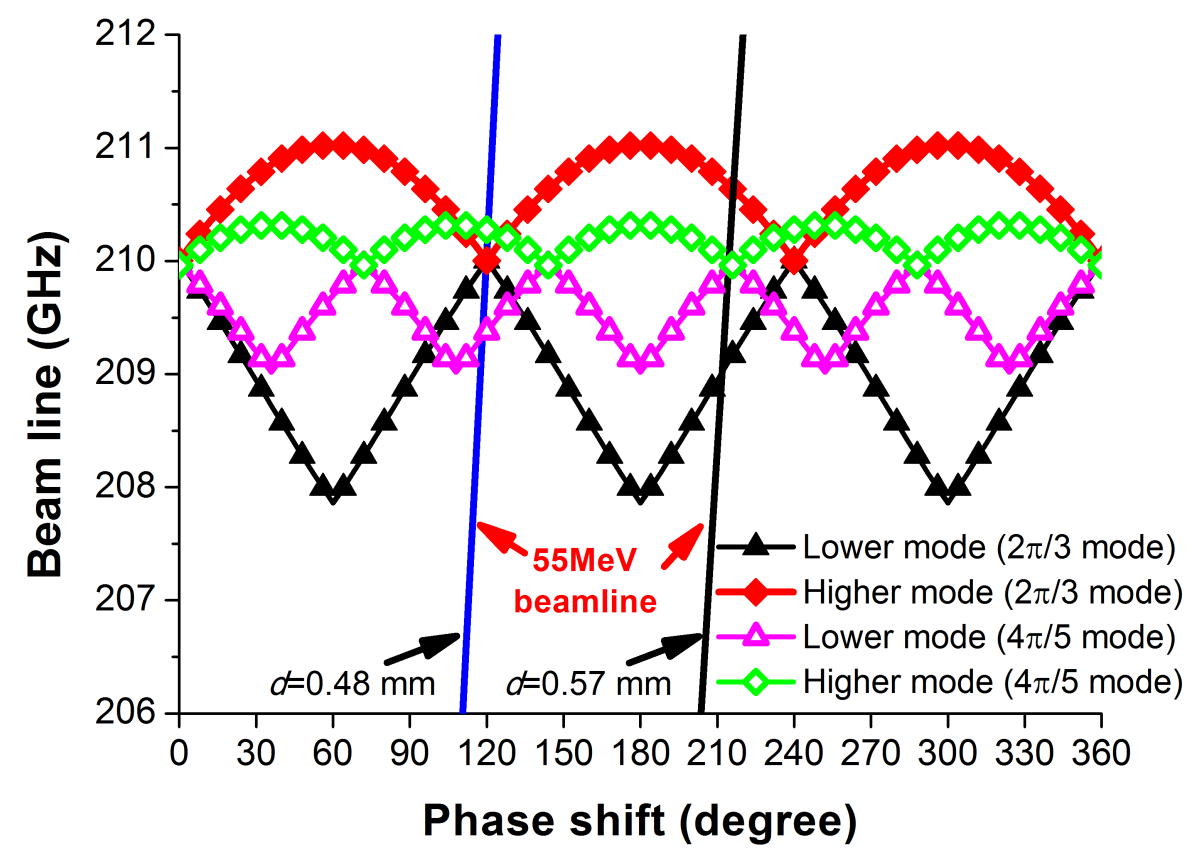

Fig. 5 Dispersion curves of the acceleration structures operating at $2 \pi / 3$ and $4 \pi / 5$ modes.

$14 \%$ decrease in the shunt impedance. Therefore a larger chamfer radius is not desirable. A chamfer radius of $0.15 \mathrm{~mm}$ is reasonable, enabling a $0.3 \mathrm{~mm}$-diameter ball-end milling tool to be used in its manufacture.

The effect on the alignment of both plates was also investigated. The structure allowed reasonably large tolerance in assembly. A $60 \mu \mathrm{m}$ offset gave a $0.5 \mathrm{GHz}$ offset in the eigenfrequency and no significant differences in shunt impedance.

The designed metallic structure is aimed to be machined by an in-house CNC mill. It has a tolerance of $10 \mu \mathrm{m}$ and the smallest high-speed milling tool available was $0.2 \mathrm{~mm}$ in diameter. The structure has the advantage of tunability by adjusting the gap distance between the two plates. It has a similar sensitivity with dimension $b$. Reducing the gap distance from $0.3 \mathrm{~mm}$ to $0.2 \mathrm{~mm}$ will result in about $7 \mathrm{GHz}$ upshift of the eigenfrequency. It is possible to tune the gap distance to achieve the required operating frequency by using a motorized stage.

\section{DISPERSION CURVE AND CHARACTERISTIC PARAMETERS}

The dispersion curves of both structures are shown in Fig. 5. The interaction frequency of the acceleration structure with a $55 \mathrm{MeV}$ electron beam is about 210 $\mathrm{GHz}$, which agrees with the analytical equation.

A summary of the characteristic parameters of the acceleration structure is shown in Table 3. Among the parameters, $\kappa$ is the loss factor which represents the amount of beam energy lost to the operating mode. It is usually normalized to per 
unit charge and per unit length of the acceleration structure and written as [16]

$$
\kappa=\frac{R_{s} P_{d}}{4 U\left(1-v_{g} / c\right)}
$$

$P_{d}$ and $U$ are the dissipated power and the stored energy in the simulated structure. They can be obtained from the results of the eigenmode solver. $v_{g}$ is the group velocity at the operating frequency, which can be obtained from the dispersion curve.

\begin{tabular}{lll}
\hline Parameter name & $\begin{array}{l}\text { structure operating } \\
\text { at } 2 \pi / 3 \text { mode }\end{array}$ & $\begin{array}{l}\text { structure operating } \\
\text { at } 4 \pi / 5 \text { mode }\end{array}$ \\
\hline Gap distance $(\mathrm{mm})$ & 0.3 & 0.3 \\
\hline Operating frequency $(\mathrm{GHz})$ & 210 & 210 \\
\hline Group velocity $(1 / c)$ & 0.02 & 0.01 \\
\hline Quality factor $Q$ & 1044.9 & 1415.3 \\
\hline Shunt impedance $R_{s}(\mathrm{M} \Omega / \mathrm{m})$ & 72.1 & 96.9 \\
\hline Loss factor $\kappa(\mathrm{nC} \cdot \mathrm{M} \Omega / \mathrm{m})$ & 23.2 & 22.8 \\
\hline
\end{tabular}

Table 3 The characteristic parameters of the acceleration structure

\section{Wakefield Simulations}

The Wakefield generated by a short electron bunch can be estimated as $[17,18]$

$$
E_{w}=2 \kappa q F\left(\sigma_{z}\right)
$$

where $F\left(\sigma_{z}\right)=e^{-\left(k_{z} \sigma_{z}\right)^{2} / 2}$ is a forming factor, $q$ and $\sigma_{z}$ are the charge of the electron bunch and the RMS bunch length, which is also defined in Table 1.

The Wakefield power can be expressed as

$$
P_{w}=\frac{E_{w}^{2} v_{g}}{4 \kappa\left(1-v_{g} / c\right)}
$$

The pulse duration of the wakefield is

$$
\tau=N d\left(1 / v_{g}-1 / c\right)
$$

where $N$ is the period number in the whole acceleration structure. The acceleration gradient is proportional to the charge in the bunch. As the loss factors for both $2 \pi / 3$ and $4 \pi / 5$ modes were similar from the eigenmode solver, the acceleration gradient cacluated from Eq. 3 would have similar values. With the CLARA beam parameters, the maximum acceleration gradient was $11.6 \mathrm{MV} / \mathrm{m}$.

More accurate acceleration gradient can be obtained using time-domain simulations. A full structure contained 105 periods, and two couplers at both ends were simulated, as shown in Fig. 6.

The electromagnetic wave was cut off at the edge of the two metallic plates and the power leakage was small because the gap between them was small. The coupler design, therefore, could be simplified to the model shown in Fig. 7(a), which can be analyzed by the aperture coupling method [19]. Each coupler contained two 


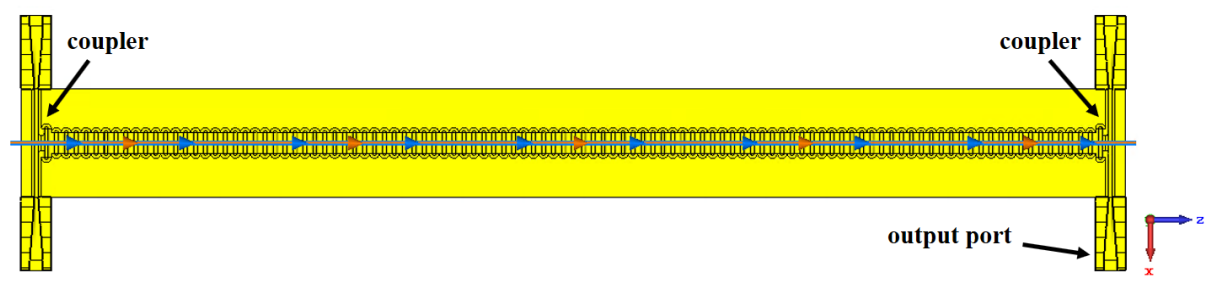

Fig. 6 Simulated structure in CST Wakefield solver.

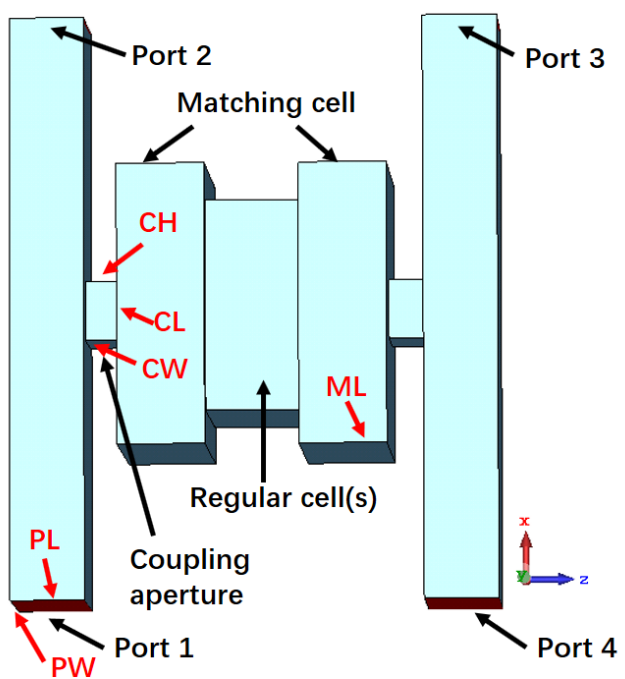

(a)

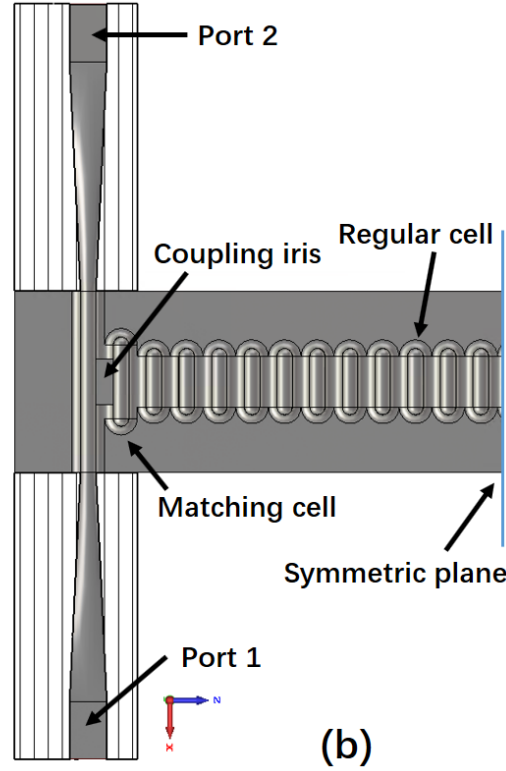

(b)

Fig. 7 (a) Simplified coupler structure, (b) coupler structure for the metallic acceleration structure.

ports at both sides and the microwave radiation was coupled in to, or out from, the regular cells through a waveguide aperture. A matching cell was used between the coupling aperture and the regular cells to provide a transitional impedance, as well to improve the bandwidth performance. From the theory, the width of the input port (PW in Fig. 7(a)) mainly controlled the operating frequency. The gap distance of the two plates also affected the operating frequency, it was fixed at 0.3 $\mathrm{mm}$. The most sensitive dimensions were the length and height of the coupling aperture (CL and $\mathrm{CH}$ ). The width of the coupling aperture (CW) mainly affected the coupling strength. The smaller its value was, the better coupling could be achieved. To simplify the machining, it was set at twice of the chamfer radius and the length of the matching cell (ML) was set to be the same as the period of the regular cells.

The optimized parameter ranges of the coupler dimensions were firstly obtained from the simplified model. Then the dimensions were used in in the metallic acceleration structure, as shown in Fig. 7(b), to be further tuned, as the effect from 
the chamfers has to be taken into account. In the simulation, a reduced period number of 15 was used to save the computing time.

The transmission results of the acceleration structure with optimal coupler dimensions are shown in Fig. 8(a). The transmission coefficient between port 1 and 3 (or 4 ) is between -5 and $-10 \mathrm{~dB}$ in the frequency range of $207.2 \mathrm{GHz}$ to $210.2 \mathrm{GHz}$ when the gap distance is fixed at $0.3 \mathrm{~mm}$. The coupler allows a certain bandwidth which is useful for cold testing the acceleration structure using a vector network analyzer (VNA). By adjusting the gap distance, the center frequency and bandwidth can be adjusted by varying the gap distance. The center frequency becomes higher and the bandwidth becomes narrower when the gap distance is reduced. Fig. 8(b) shows the field pattern of the coupler at $210 \mathrm{GHz}$ when the gap distance is $0.3 \mathrm{~mm}$.

In this study, the time evolution of the generated wakefield was verified by using the Wakefield solver in CST particle studio. A few field probes were placed at the beam path to diagnose the acceleration gradient. The generated wakefield can also be extracted out from the couplers at both ends. The simulation results of the Wakefield strength generated by one single bunch are shown in Fig. 9 . The $4 \pi / 5$ mode reached the peak field strength of close to $20 \mathrm{MV} / \mathrm{m}$ while the $2 \pi / 3$ mode is about $17 \mathrm{MV} / \mathrm{m}$. The $4 \pi / 5$ mode has a much longer tail which was mainly caused by the lower group velocity. The average amplitudes of the acceleration gradient from the Wakefield simulations were close to the predicted value from the equations.

The acceleration gradient generated by one single bunch was relatively low and mainly due to the magnitude of the bunch charge of the CLARA beam. One way to increase the acceleration gradient is to excite the acceleration structure with an electron bunch train. With proper time sequencing, the Wakefield excited by an individual bunch can be added up by using a train of bunches resulting in a higher accelerating gradient. The operating frequency of the RF photo-injector gun is $3 \mathrm{GHz}$, which corresponds to a time interval between the bunch of $0.33 \mathrm{~ns}$. The Wakefield strength driven by such a bunch train was also simulated. Fig. 10 shows that the Wakefield strength can reach about $100 \mathrm{MV} / \mathrm{m}$ when the bunch train contains 10 bunches.

\section{CONCLUSION}

In this paper, the design of a metallic Wakefield acceleration structure operating at $210 \mathrm{GHz}$ is presented. Both the structures operating at $2 \pi / 3$ mode and $4 \pi / 5$ mode were investigated and compared. The $4 \pi / 5$ mode is less sensitive to the period of the structure, as well as having a higher impedance. In wakefield simulation by CST particle studio, the peak field strength of $20 \mathrm{MeV}$ was achieved based on the single electron bunch parameters of CLARA. It was mainly limited by the charge contained in a single electron bunch. Further improvement in the acceleration gradient can be realized using a bunch train. In the simulation, the acceleration gradient of $100 \mathrm{MV} / \mathrm{m}$ was achieved by using a 10-bunch train.

A metallic structure operating at $210 \mathrm{GHz}$ and $4 \pi / 5$ mode will be manufactured at the University of Strathclyde. Computer Numerical Controlled machining using an air driven high speed (40,000 rpm) turbine will be used. Its microwave properties will be measured using a vector network analyzer. An experiment is proposed to 


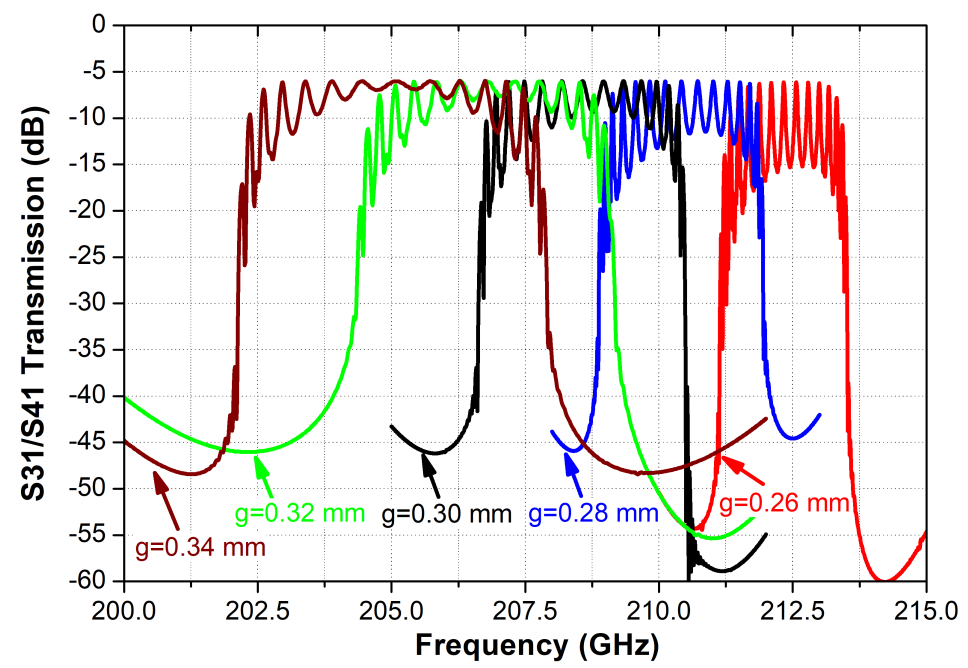

(a)

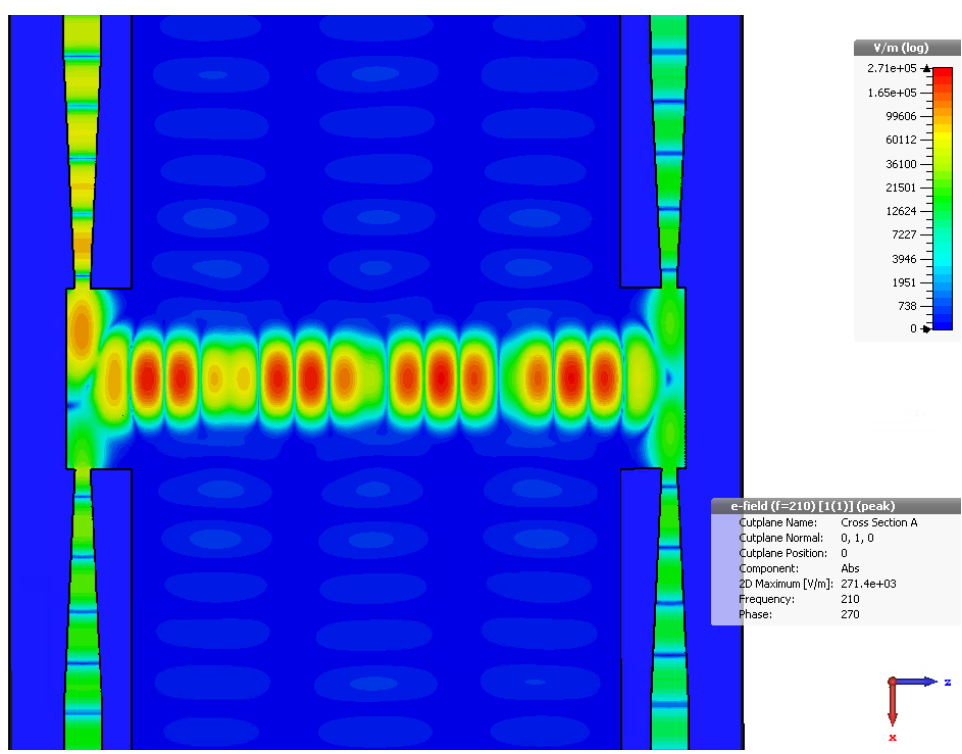

(b)

Fig. 8 (a) Transmission coefficients of the metallic acceleration structure with the coupler at different gap distance, (b) field pattern at $210 \mathrm{GHz}$ when the gap distance is $0.3 \mathrm{~mm}$.

be carried out using the CLARA facility at the Daresbury Laboratory. A single bunch measurement on CLARA is proposed to verify the design. Future work will involve the study of electron beam dynamics through the planar metallic structure. 


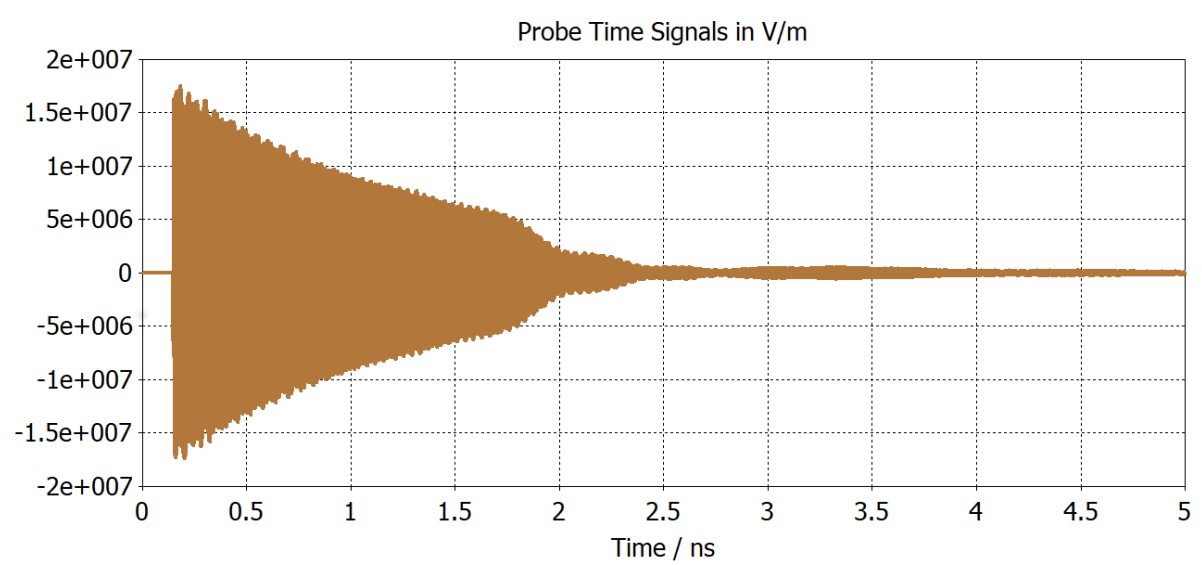

(a)

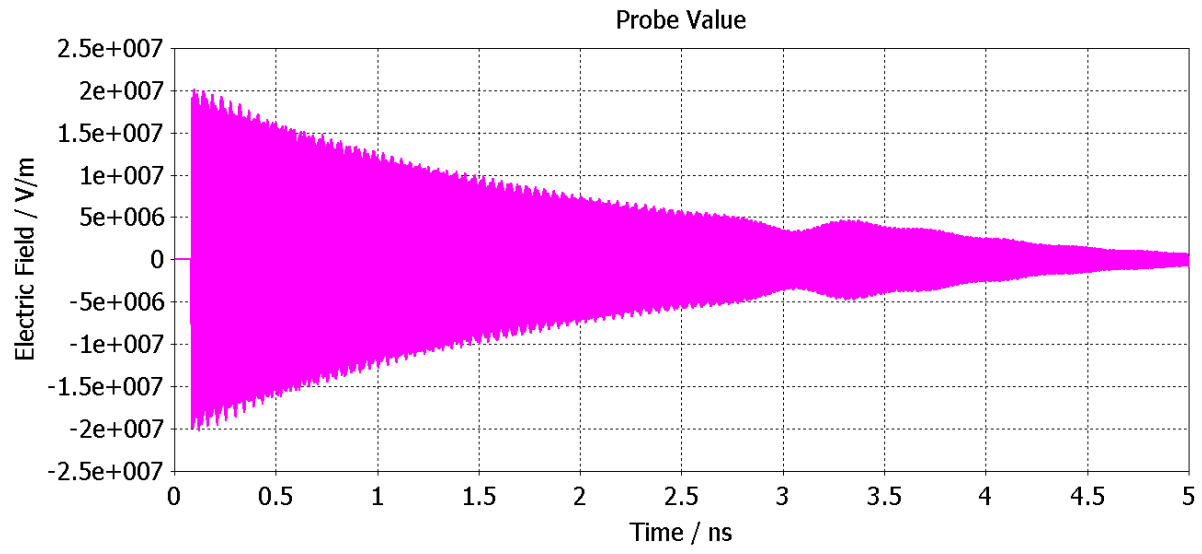

(b)

Fig. 9 Wakefield simulation results with a single bunch (a) $2 \pi / 3$ mode and (b) $4 \pi / 5$ mode.

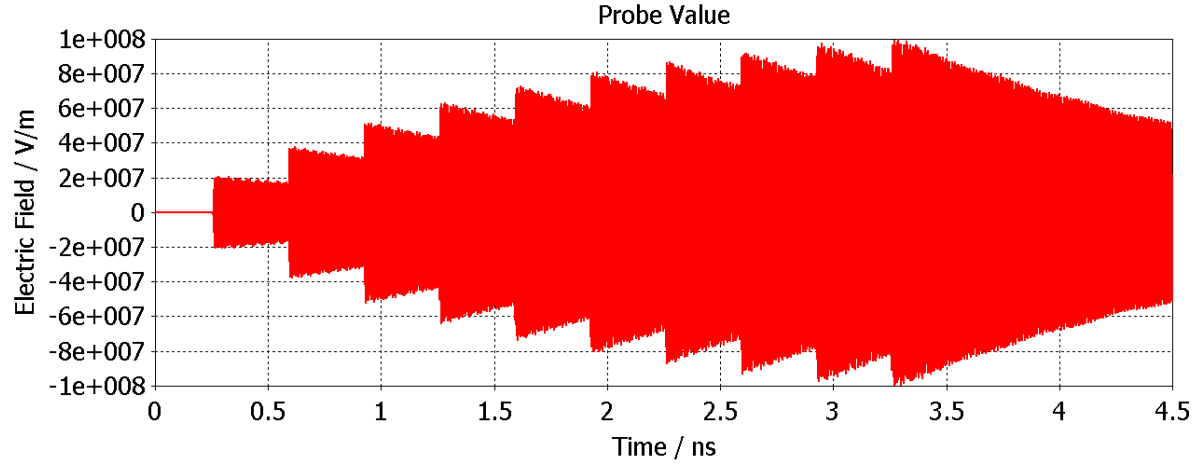

Fig. 10 Wakefield simulation result with a bunch train contains 10 bunch. 
Acknowledgements The authors would like to thank the Dr. Yuri Saveliv of ASTeC and the Cockcroft Institute for the useful discussion on the CLARA test facility at Daresbury Laboratory, UK.

\section{References}

1. R. Wilson, Science 164(3880), 674 (1969). DOI 10.1126/science.164.3880.674-a

2. T. Raubenheimer, C. Adolphsen, D. Burke, P. Chen, S. Ecklund, J. Irwin, G. Loew, T. Markiewicz, R. Miller, E. Paterson, N. Phinney, K. Ross, R. Ruth, J. Sheppard, H. Tang, K. Thompson, P. Wilson, in Proceedings Particle Accelerator Conference, vol. 2 (1995), vol. 2, pp. 698-700. DOI 10.1109/PAC.1995.504762

3. A. De Roeck, J. Ellis, C. Grojean, S. Heinemeyer, K. Jakobs, G. Weiglein, J. Wells, G. Azuelos, S. Dawson, B. Gripaios, T. Han, J. Hewett, M. Lancaster, C. Mariotti, F. Moortgat, G. Moortgat-Pick, G. Polesello, S. Riemann, M. Schumacher, K. Assamagan, P. Bechtle, M. Carena, G. Chachamis, K.F. Chen, S. De Curtis, K. Desch, M. Dittmar, H. Dreiner, M. Dührssen, B. Foster, M.T. Frandsen, A. Giammanco, R. Godbole, S. Gopalakrishna, P. Govoni, J. Gunion, W. Hollik, W.S. Hou, G. Isidori, A. Juste, J. Kalinowski, A. Korytov, E. Kou, S. Kraml, M. Krawczyk, A. Martin, D. Milstead, V. Morton-Thurtle, K. Moenig, B. Mele, E. Ozcan, M. Pieri, T. Plehn, L. Reina, E. RichterWas, T. Rizzo, K. Rolbiecki, F. Sannino, M. Schram, J. Smillie, S. Sultansoy, J. Tattersall, P. Uwer, B. Webber, P. Wienemann, The European Physical Journal C 66(3), 525 (2010). DOI $10.1140 /$ epjc/s10052-010-1244-3

4. F.V. Hartemann, F. Albert, Technical Report LLNL-TR-416320 (2009). DOI $10.2172 / 967742$

5. T. Sakurai, H. Ego, T. Inagaki, T. Asaka, D. Suzuki, S. Miura, Y. Otake, Phys. Rev. Accel. Beams 20, 042003 (2017). DOI 10.1103/PhysRevAccelBeams.20.042003. URL https://link.aps.org/doi/10.1103/PhysRevAccelBeams.20.042003

6. T. Argyropoulos, N. Catalan-Lasheras, A. Grudiev, G. Mcmonagle, E. Rodriguez-Castro, I. Syrachev, R. Wegner, B. Woolley, W. Wuensch, H. Zha, V. Dolgashev, G. Bowden, A. Haase, T.G. Lucas, M. Volpi, D. Esperante-Pereira, R. Rajamäki, Phys. Rev. Accel. Beams 21, 061001 (2018). DOI 10.1103/PhysRevAccelBeams.21.061001. URL https://link.aps.org/doi/10.1103/PhysRevAccelBeams.21.061001

7. S.M. Hooker, Nature Photon. 7, 775 (2013). DOI 10.1038/nphoton.2013.234

8. E.A. Peralta, K. Soong, R.J. England, E.R. Colby, Z. Wu, B. Montazeri, C. McGuinness, J. McNeur, K.J. Leedle, D. Walz, E.B. Sozer, B. Cowan, B. Schwartz, G. Travish, R.L. Byer, Nature 503, 91 EP (2013)

9. S. Jamison, et al., in Proceedings, 28th International Linear Accelerator Conference (LINAC16): East Lansing, Michigan, September 25-30, 2016 (2017), p. MOOP09. DOI 10.18429/JACoW-LINAC2016-MOOP09

10. J.A. Clarke, D. Angal-Kalinin, N. Bliss, R. Buckley, S. Buckley, R. Cash, P. Corlett, L. Cowie, G. Cox, G.P. Diakun, D.J. Dunning, B.D. Fell, A. Gallagher, P. Goudket, A.R. Goulden, D.M.P. Holland, S.P. Jamison, J.K. Jones, A.S. Kalinin, W. Liggins, L. Ma, K.B. Marinov, B. Martlew, P.A. McIntosh, J.W. McKenzie, K.J. Middleman, B.L. Militsyn, A.J. Moss, B.D. Muratori, M.D. Roper, R. Santer, Y. Saveliev, E. Snedden, R.J. Smith, S.L. Smith, M. Surman, T. Thakker, N.R. Thompson, R. Valizadeh, A.E. Wheelhouse, P.H. Williams, R. Bartolini, I. Martin, R. Barlow, A. Kolano, G. Burt, S. Chattopadhyay, D. Newton, A. Wolski, R.B. Appleby, H.L. Owen, M. Serluca, G. Xia, S. Boogert, A. Lyapin, L. Campbell, B.W.J. McNeil, V.V. Paramonov, Journal of Instrumentation 9(05), T05001 (2014)

11. P. McIntosh, et al., in Proceedings, 5th International Particle Accelerator Conference (IPAC 2014): Dresden, Germany, June 15-20, 2014 (2014), p. WEPME083

12. M. Dal Forno, V. Dolgashev, G. Bowden, C. Clarke, M. Hogan, D. McCormick, A. Novokhatski, B. O'Shea, B. Spataro, S. Weathersby, S.G. Tantawi, Phys. Rev. Accel. Beams 19, 111301 (2016). DOI 10.1103/PhysRevAccelBeams.19.111301

13. M. Dal Forno, V. Dolgashev, G. Bowden, C. Clarke, M. Hogan, D. McCormick, A. Novokhatski, B. Spataro, S. Weathersby, S.G. Tantawi, Phys. Rev. Accel. Beams 19, 051302 (2016). DOI 10.1103/PhysRevAccelBeams.19.051302

14. D. Wang, S. Antipov, C. Jing, J.G. Power, M. Conde, E. Wisniewski, W. Liu, J. Qiu, G. Ha, V. Dolgashev, C. Tang, W. Gai, Phys. Rev. Lett. 116, 054801 (2016). DOI 10.1103/PhysRevLett.116.054801 
15. C. Corp., CST STUDIO SUITE, www.cst.com

16. K.L.F. Bane, G. Stupakov, Phys. Rev. ST Accel. Beams 6, 024401 (2003). DOI 10.1103/PhysRevSTAB.6.024401

17. M.D. Forno, V. Dolgashev, G. Bowden, C. Clarke, M. Hogan, D. McCormick, A. Novokhatski, B. O'Shea, B. Spataro, S. Weathersby, S.G. Tantawi, Nuclear Instruments and Methods in Physics Research Section A: Accelerators, Spectrometers, Detectors and Associated Equipment 864, 12 (2017). DOI https://doi.org/10.1016/j.nima.2017.05.014

18. F. Gao, M.E. Conde, W. Gai, C. Jing, R. Konecny, W. Liu, J.G. Power, T. Wong, Z. Yusof, Phys. Rev. ST Accel. Beams 11, 041301 (2008). DOI 10.1103/PhysRevSTAB.11.041301

19. D.M. Pozar, Microwave engineering (Fourth edition. Hoboken, NJ, Wiley, 2012) 